\title{
Coping Style Assessment Tool
}

National Cancer Institute

\section{Source}

National Cancer Institute. Coping Style Assessment Tool. NCI Thesaurus. Code C93220.

A self-report inventory that measures how a person copes with problems and stress. The protocol includes 8 scales within 2 categories. (Copyright Psychological Assessment Resources) 